\title{
Phenotypic evaluation of segregant population derived by crossing table grape varieties
}

\author{
L.R. Forleo, A. L'Abbate, C. Bergamini, M. Velenosi, A.D. Marsico, M.F. Cardone, D. Antonacci, R. Velasco \\ e R. Perniola
}

Research Centre for Viticolture and Enology (CREA - VE), Via Casamassima 148, 70010 Turi-Bari, Italy

\begin{abstract}
Sintesi. In the CREA-Viticoltura ed Enologia, Lab of Turi, during the last ten years, we started a breeding program for table grapes to obtain new seedless varieties, by using conventional breeding by crossings, and embryo rescue techniques. Other than seedlessness, additional targets for this breeding program are: the possibility of extending the harvesting period, the attitude to cold storage, transport and shelf-life, resistance to diseases both on the plant and in post-harvest conditions, the good productivity, the quality of the grapes, the easy cultivation management of the vineyard (reduced water, nutritional requirements, etc ...). More than 10.000 new genotypes have been obtained through the use of over 20 table grape varieties and more than 18 different crossing combinations. The following characteristics have been observed on these individuals for more than three years: berry color, length and weight cluster, average berry weight, sugars, $\mathrm{pH}$, acidity, class of seedlessness, floral morphology, resistance to diseases. The results of these activities revealed that some combinations have better performance among all. In the near future, this activity will allow to focus on parental genotypes able to provide individuals with the best desired traits.
\end{abstract}

\section{Introduzione}

L'Italia, con una produzione di circa 1.2 milioni di tonnellate di uva da tavola, si posiziona al sesto posto come paese produttore a livello mondiale dopo Cina, USA, Iran, Turchia ed Egitto [1]. Il 60\% circa dell'uva da tavola italiana viene prodotta in Puglia. Le varietà maggiormente coltivate sono con semi: Italia, Victoria e Red Globe; negli ultimi anni si è assistito ad uno spiccato interesse verso la coltivazione di varietà apirene, la cui origine è quasi sempre extra UE. Per il futuro si prevede una graduale sostituzione delle varietà con semi con quelle apirene, che rispondono maggiormente alle esigenze di mercato (soprattutto del Nord-Europa che rappresenta per l'Italia un mercato molto importante) e si prestano meglio nella preparazione di prodotti alimentari.

Purtroppo, molte varietà apirene diffuse sul mercato presentano problemi di ambientamento nella nostra regione, in quanto ottenute in Paesi esteri caratterizzati da condizioni pedoclimatiche differenti. A tal fine, si rende necessario costituire nuove varietà con l'obiettivo di ottenere uve apirene adatte ai nostri ambienti.

\subsection{Programma di breeding presso il Crea}

Il progetto di miglioramento genetico delle uve da tavola presso il CREA-VE, sede di Turi, ha avuto inizio nel 2008. L'obiettivo principale è stato l'ottenimento di varietà di uve da tavola apirene attraverso tecniche convenzionali e non, come l'embryo rescue, che permette di utilizzare negli incroci due varietà apirene come genitori. La scelta di indirizzare la ricerca sull'ottenimento di nuove varietà apirene è dettata dalla necessità, da parte delle imprese, di rimanere competitive sui mercati nazionali ed internazionali, ma anche di avere il "controllo" delle stesse, al fine di impostare precise politiche commerciali, indispensabili per garantire stabilità e prospettiva di reddito ai produttori di uva da tavola italiani.

A queste nuove varietà si è chiesto che fossero anche riconoscibili per tipicità e richiamo del territorio. Tale necessità deriva dalla difficoltà di adattamento delle varietà di uve da tavola estere agli areali di coltivazione italiani. Gli obiettivi del miglioramento genetico del nostro centro, per la selezione di nuove varietà di uve da tavola, hanno riguardato caratteri specifici, in particolare: la possibilità di estensione del periodo di raccolta, l'attitudine alla frigoconservazione, al trasporto e alla shelf life, la resistenza a malattie, sia sulla pianta che in postraccolta, la buona produttività, la produzione di acini senza semi e dalle dimensioni elevate, la facile gestione colturale del vigneto (ridotta richiesta idrica, nutrizionale, ecc...). Questa attività ha suscitato per la prima volta in Italia il forte interesse di privati, i quali hanno fondato l'associazione di produttori NuVaUT, che ha stretto con il CREA un accordo formalizzatosi nel corso del 2018 per lo sviluppo e la sperimentazione presso le stesse aziende delle migliori 100 varietà costituite.

\section{Materiali e metodi}

L'attività di ricerca è stata svolta presso le aziende sperimentali di proprietà del CREA-VE: "Lamarossa" in agro di Rutigliano e "Paparusso" in agro di Turi. Oltre alle collezioni già presenti è stato avviato un programma di recupero di varietà di uve da tavola diffuse sul territorio nazionale, con particolare riferimento alle regioni del Meridione d'Italia. Le varietà recuperate e conservate presso l'azienda sperimentale "Lamarossa" sono state 
oggetto di studio per la verifica di sinonimie ed omonimie attraverso analisi genetica molecolare e quindi valutate per le caratteristiche qualitative e produttive delle uve. Da queste, nel periodo 2008-2012, sono state selezionate oltre 20 varietà, realizzando più di 18 combinazioni di incrocio e ottenendo più di 10.000 nuovi individui. Le attività di miglioramento genetico oltre alle fasi di individuazione delle varietà parentali, selezione delle infiorescenze e realizzazione degli incroci, hanno previsto una delicata attività di recupero dei semi e degli embrioni, fino all'ottenimento e trasferimento in campo delle piantine. Nel 2013 si sono avuti i primi frutti, sui quali sono stati effettuati rilievi quanti-qualitativi. Delle differenti popolazioni segreganti ottenute, di particolare interesse è risultata quella derivante dall'incrocio di una varietà a bacca rossa con seme (var A) con una a bacca bianca apirena (var B). L'analisi dei dati ha evidenziato come tale combinazione abbia dato le performance migliori, da cui sono stati individuati nuovi genotipi di interesse per l'ottenimento di nuove varietà di uva da tavola.

\subsection{Ottenimento nuova progenie}

\subsubsection{Caratteristiche dei parentali}

\section{Genitore femminile (var A)}

Foglia adulta: pentagonale, con tre o cinque lobi, seno peziolare aperto, sagomato a $\mathrm{U}$ o a $\mathrm{V}$.

Grappolo: dalle dimensioni elevate (lungo $28 \mathrm{~cm}$ ), di forma conica con peso medio di circa $1200 \mathrm{~g}$, mediamente compatto, con 1-2 ali.

Acino: di grande dimensione del peso di circa $13.5 \mathrm{~g}$, sferoidale, buccia dal colore rosso, polpa incolore dal sapore neutro; presenta 3-4 vinaccioli per acino.

Epoca di germogliamento: tardiva (III decade di aprile).

Epoca di fioritura: tardiva (II decade di giugno).

Epoca di maturazione: medio-tardiva (II-III decade di settembre, fino a dicembre se coperta con teli di copertura).

\section{Genitore maschile (var B)}

Foglia adulta: orbicolare, con cinque lobi, seno peziolare aperto sagomato a V.

Grappolo: di medio-grandi dimensioni (lungo $27 \mathrm{~cm}$ ), dalla forma cilindrico-conica con peso medio di circa $1042 \mathrm{~g}$, alato, mediamente compatto.

Acino: di dimensioni grandi $(8.8 \mathrm{~g})$, dalla forma ellissoidale stretta, buccia di colore verde-giallo, spessa; polpa croccante dal sapore neutro; apirena ma con semi leggermente distinguibili.

Epoca di germogliamento: tardiva (III decade di aprile).

Epoca di fioritura: media (I decade di giugno).

Epoca di maturazione: media, medio tardiva (I-II decade di settembre).

\subsubsection{Incrocio tra varietà $A$ con seme e varietà $B$ apirena}

Le infiorescenze, della varietà portaseme (var A) selezionata, sono state demasculate (prima della fioritura) con l'ausilio di pinzette e subito dopo isolate dall'ambiente circostante con sacchetti di carta per evitare l'inquinamento da parte di polline estraneo. Alla comparsa del liquido stigmatico, si è proceduto alla fecondazione con il polline della varietà scelta come genitore maschile (var B). Dopo l'allegagione i grappoli sono stati liberati dai sacchetti e sono stati lasciati sulla pianta fino a maturazione fisiologica. Dopo la raccolta i grappoli sono stati conservati in cella frigorifero alla temperatura di circa $+4{ }^{\circ} \mathrm{C}$ fino all'estrazione dei semi.

L'estrazione dei semi è avvenuta tra l'ultima decade di settembre e la prima di ottobre; dopo aver estratto i semi dalle bacche si è proceduto alla loro sterilizzazione e successiva asciugatura a temperatura ambiente.

Successivamente, poiché il seme presenta dormienza dell'embrione condizionata da fattori ormonali, per ottenere una buona percentuale di germinazione i semi sono stati conservati per un periodo di circa 6 mesi ad una temperatura di circa $+4{ }^{\circ} \mathrm{C}[2]$.

\subsubsection{Germinazione semi e piantamento in campo}

La semina è stata effettuata nella primavera successiva in dischetti di torba precompressa a questa ha fatto seguito il rinvaso e l'allevamento in serra. A marzo dell'anno successivo le piantine ormai ben lignificate sono state messe a dimora in piena terra, nel campo dell'azienda sperimentale "Paparusso" in agro di Turi.

Il sistema di allevamento è stato impostato a tendone con un sesto d'impianto di $2.3 \times 0.4 \mathrm{~m}$ e in regime irriguo di $2.700 \mathrm{~m}^{3} /$ ha/anno.

\subsection{Analisi molecolari e fenotipiche}

\subsubsection{MAS}

Il programma di miglioramento genetico si è avvalso della selezione assistita da marcatori (Marker Assisted Selection-MAS). Dopo l'estrazione del DNA, da un campione di foglia e successiva amplificazione PCR, si è proceduti all'analisi di regioni del genoma della vite collegate a specifici caratteri d'interesse. L'accertamento della paternità desiderata nell'incrocio e la stenospermocarpia, utilizzando rispettivamente tre marcatori SSR e il marcatore p3_VvAGL11, sono stati i primi caratteri analizzati. Con tali tecniche si è pertanto in grado di velocizzare ed anticipare di almeno un quinquennio le fasi di selezione e studio dei nuovi materiali genetici finalizzati a creare moderne varietà, rispetto alle procedure tradizionali.

\subsubsection{Rilievi quanti-qualitativi}

Nel quadriennio 2013-2016 sono stati effettuati rilievi finalizzati ad accertare la risposta quantitativa (lunghezza grappolo, peso medio grappolo, peso medio bacca, ${ }^{\circ}$ Brix, $\mathrm{pH}$, acidità totale) e qualitativa (colore, classe apirenia) della produzione. Le determinazioni chimiche sono state eseguite secondo i metodi ufficiali (G.U.C.E. n. 272 del 03.10.1990). Le analisi statistiche (test del Chi quadro e correlazione di Spearman) sono state effettuate mediante l'utilizzo di R (versione 3.4.4).

\section{Risultati e discussione}

In Fig. 1 è riportata la distribuzione del colore negli individui osservati, su 456 nuovi genotipi 216 presentano il colore bianco derivante dal parentale maschile e 241 hanno la bacca colorata derivante dal parentale femminile, questi 


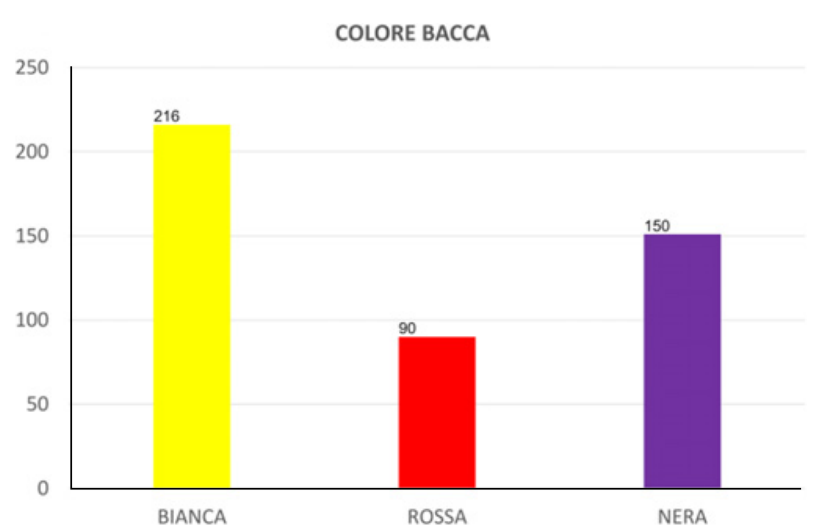

Figura 1. Distribuzione del colore.

ultimi sono così distribuiti: 90 a bacca rossa e 150 a bacca nera.

La distribuzione attesa del carattere colore bacca prevede un rapporto di 1:1 tra gli individui a bacca bianca e quelli colorati. Il test del $\chi^{2}$ fornisce un valore pari a 0.24 pertanto la distribuzione osservata non si discosta in maniera significativa da quella attesa.

Per quanto riguarda la distribuzione delle classi di apirenia così come mostrato in Fig. 2a si hanno 256 genotipi con presenza di seme (classe 4) e 200 con apirenia (classi 1-2-3).

In questo caso il valore del del $\chi^{2}$ è pari a 0.009 , rivelando pertanto un discostamento della distribuzione osservata da quella attesa. In particolare, gli individui con seme risultano leggermente sovrarappresentati rispetto all'atteso. Tale osservazione può essere spiegata dal tipo di analisi dei dati adottata in cui la classe di apirenia assegnata a ciascuna varietà è la più alta tra tutte quelle registrate nei rilievi delle quattro annate: pertanto, questo ha causato una leggera sottostima della classe 3 a favore della classe 4.

Il peso medio acino (PMA) come da Fig. 2b risulta uguale a:

\section{$3.67 \mathrm{~g}$ per la classe 1 ;}

$3.92 \mathrm{~g}$ per la classe 2 ;

$3.56 \mathrm{~g}$ per la classe 3 ;

$5.61 \mathrm{~g}$ per la classe 4 ;

$5.39 \mathrm{~g}$ come media di tutte e quattro le classi.

In Fig. 2c si osserva la correlazione tra il peso acino e la classe di apirenia. Come atteso il PMA aumenta in funzione dello sviluppo del vinacciolo.

Nella Fig. 3 è possibile osservare come si distribuiscono i caratteri lunghezza e peso grappolo. Per quanto riguarda la lunghezza abbiamo un valore minimo pari a $8 \mathrm{~cm}$ e uno massimo di $52 \mathrm{~cm}$, mentre il valore medio è pari a $26.9 \mathrm{~cm}$, quest'ultimo comparabile con la lunghezza dei due parentali aventi $28 \mathrm{~cm}$ il portaseme e $27 \mathrm{~cm}$ l'impollinante.

Il peso medio grappolo presenta un valore di $336.7 \mathrm{~g}$ mentre i valori min e max sono rispettivamente $24 \mathrm{~g}$ e $1653.2 \mathrm{~g}$. I parentali invece hanno peso medio grappolo pari a $1200 \mathrm{~g}$ per la var A e $1042 \mathrm{~g}$ per la var B. Il valore medio risulta pertanto molto distante dal valore dei parentali ma questa differenza è da attribuirsi molto probabilmente sia alla giovane età delle piante, sia al sesto d'impianto fitto, ed infine al fatto che $i$ due genitori sono varietà selezionate mentre nella discendenza a

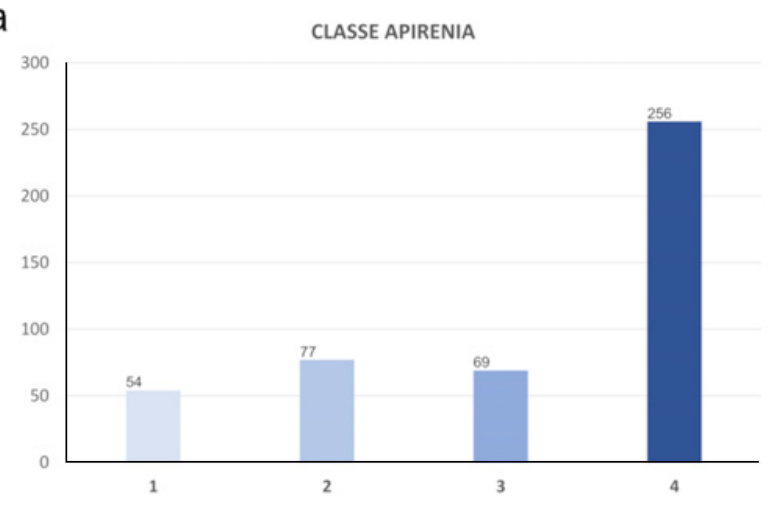

b
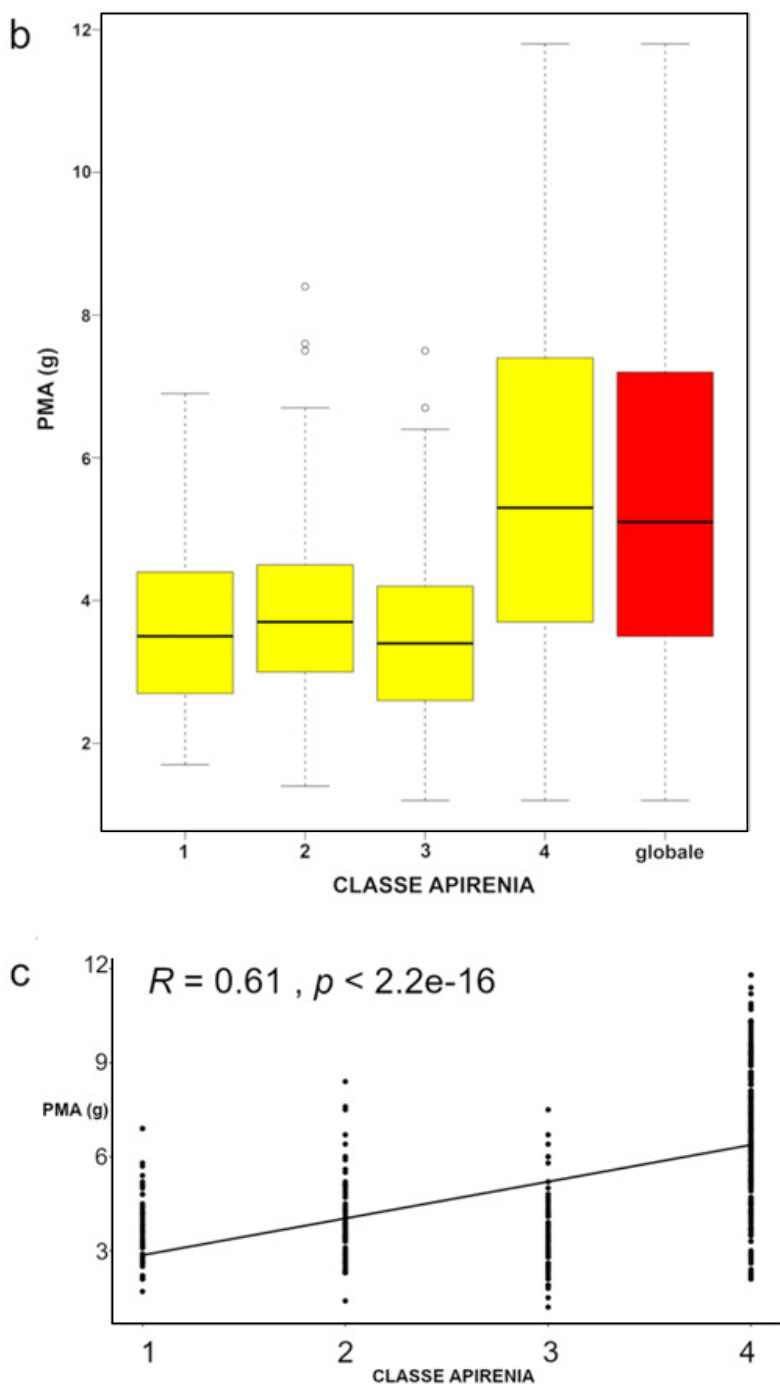

Figura 2. Distribuzione delle classi di apirenia.

sono rappresentate tutte le combinazioni geniche ottenute, anche le tante sfavorevoli per la manifestazione del carattere in questione.

In Fig. 4 è possibile osservare come il calendario di maturazione sia molto ampio, filtrando i dati sulla base del parametro minimo di commercializzazione proposto dall'OIV che ritiene valide delle uve aventi un valore compreso tra 20 e 25 del rapporto Solidi Solubili Totali e Acidità Totale, si va dal 29 luglio al 17 ottobre nel 2013, dal 10 agosto al 29 ottobre nel 2014, dal 28 luglio al 28 ottobre nel 2015, dal 2 agosto al 18 novembre del 2016. 


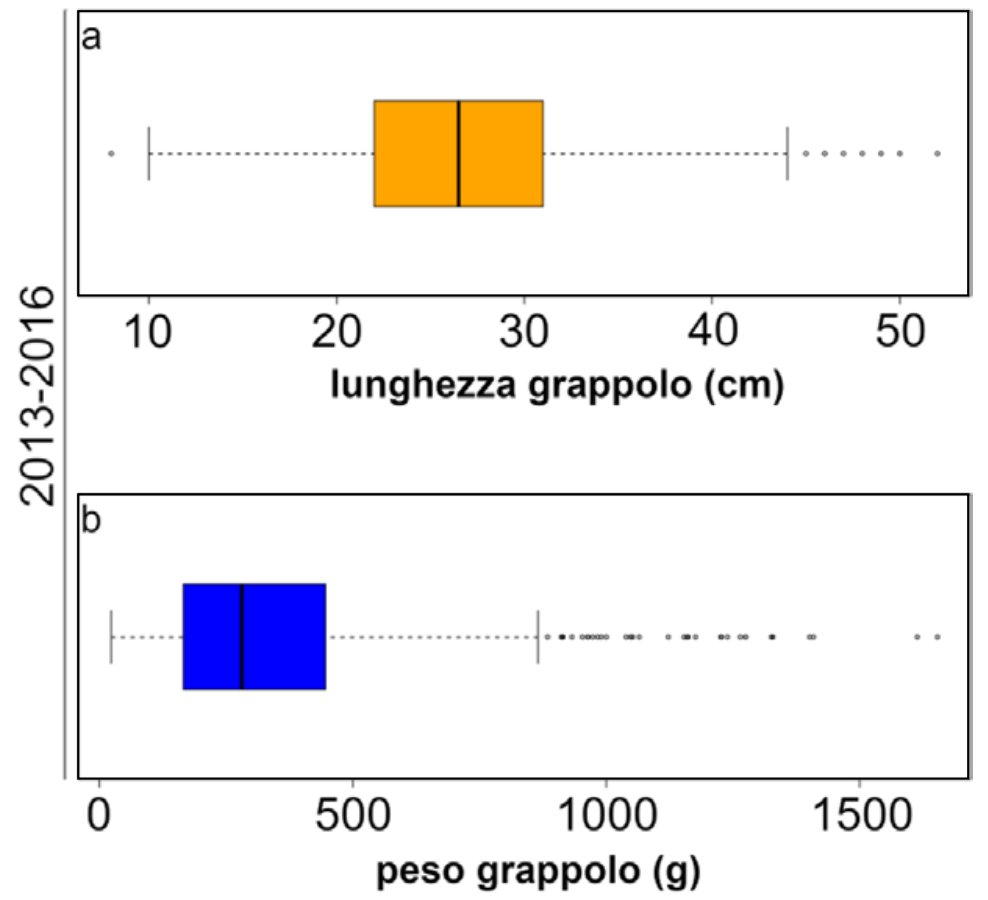

Figura 3. Distribuzione delle classi di lunghezza e peso grappolo.

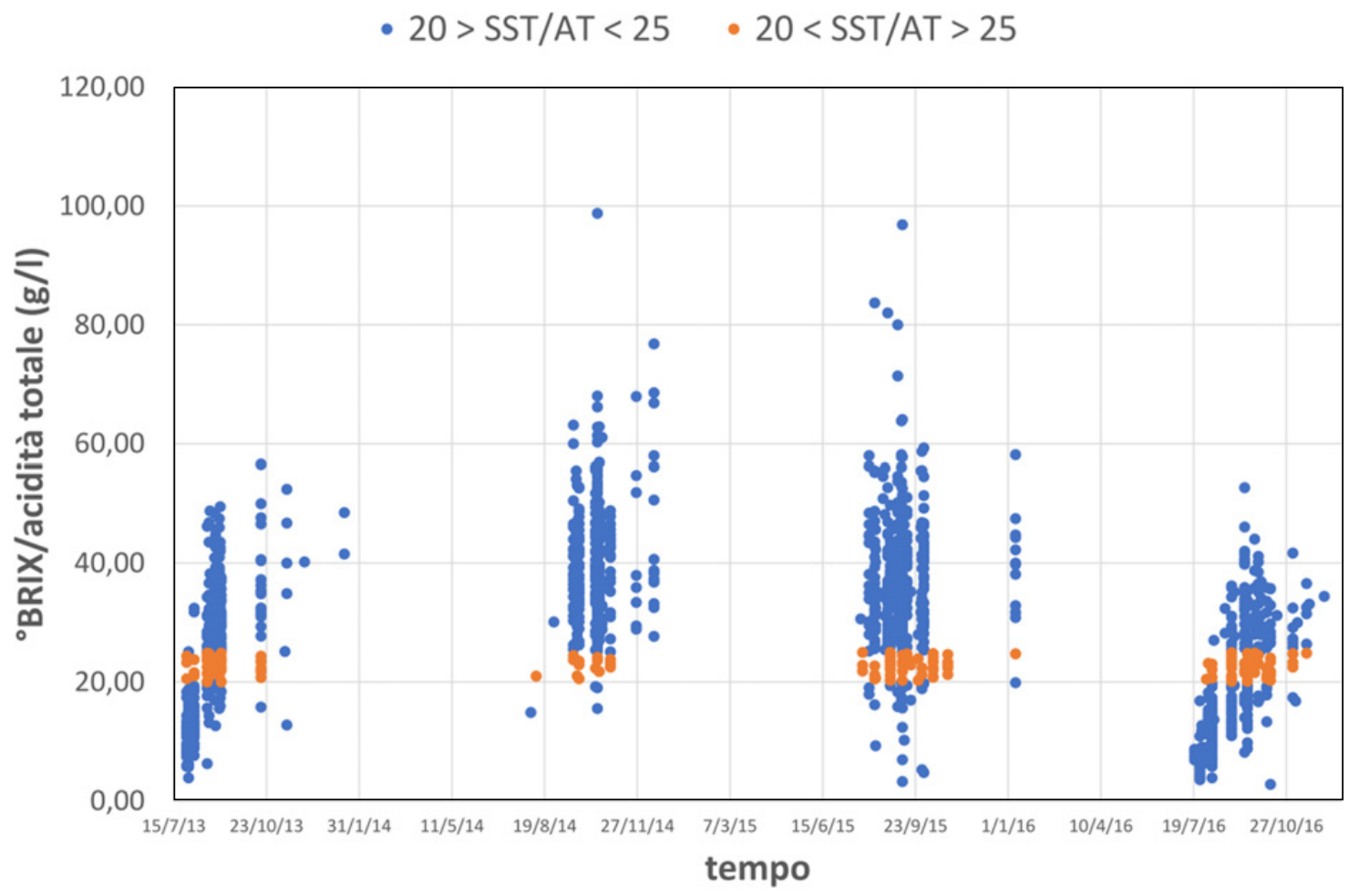

Figura 4. Distribuzione dei nuovi genotipi in base al rapporto zuccheri acidi.

Ma da una attenta lettura si evince come questo calendario possa essere più ampio, infatti in queste due date ci sono ancora genotipi i cui frutti non hanno raggiunto il valore ottimale di maturazione. Entrambi i parentali mostrano epoche di maturazione intermedia rispetto all'intervallo osservato nella progenie, rispettivamente le decadi II-III di settembre per var A e I-II decade di settembre per var B.

\section{Conclusioni}

Presso il CREA-VE di Turi nell'ultimo decennio è stato avviato un programma di breeding per il miglioramento genetico dell'uva da tavola. In tale quadro sono state create delle popolazioni di incrocio per scopi di studio, in cui sono stati conservati tutti gli individui derivati dall'incrocio di due genitori. In questo lavoro sono 
riportati i risultati dell'attività di caratterizzazione di una di tali popolazioni di incrocio. Le valutazioni ripetute nel corso di quattro annate sono state utili a definire i protocolli migliori per le valutazioni delle progenie e la selezione dei migliori genitori per il breeding.

\section{Riferimenti}

[1] AAVV. (2018) OIV Statistical Report on World Vitiviniculture: 2017 World Vitiviniculture Situation [2] H.T. Hartmann, D.E. Kester, Propagazione Delle Piante (Bologna, Edagricole, 1990) 